\title{
EFFECT OF TWO SEQUENCING COMBINATIONS OF PHYSICAL PRACTICE AND MENTAL PRACTICE ON BALANCE IN STROKE PATIENTS
}

\author{
Neha Ramchandra Tambe *1, Shamla Wamanrao Pazare ${ }^{2}$. \\ ${ }^{* 1}$ MPT (Neurosciences) Physiotherapist, Pune, Maharashtra, India. \\ ${ }^{2}$ Professor, CMF's College of Physiotherapy, Chinchwad, Pune, Maharashtra, India.
}

\section{ABSTRACT}

Aim \& Objective: To study and compare the effect of two sequencing combinations of PhysicalPractice and Mental Practice in improving Balance in stroke patients usingBalance evaluation system test and Activities specific balance confidence scale.

Method: The experimental study was conducted on 30 subjects with strokes in the age group of 45-60 years, including both genders, with duration of stroke 1-6 months, and patients with the scoring of berg balance scale in between 21-40.All the subjects were assessed for the outcome measures at baseline using BESTest scale and $A B C$ scale. Both the groups received treatment for 3 times/ week for 4 weeks. Re-assessment of all the outcome measures post 4-week intervention was done.Both the groups received conventional therapy and sequencing combinations of Mental practice and Physical practice.

Results: A significant improvement was seen in both the groups of sequencing combinations in improving balance in stroke patients.

Conclusion:The present study concludes that both the sequences ofcombinations of Mental Practice and Physical Practice are equally effective inimproving balance in stroke patients.

KEYWORDS: Motor imagery, Mental practice, Physical practice, Stroke, Balance, Sequencing.BESTest, ABC score.

Address for correspondence: Dr. Neha RamchandraTambe, MPT (Neurosciences) Physiotherapist, Pune, Maharashtra, India.E-Mail: nehatambe14@gmail.com

Access this Article online

Quick Response code

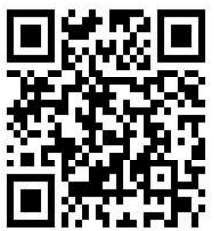

DOI: $10.16965 /$ ijpr.2020.131
Journal Information

International Journal of Physiotherapy and Research

ISSN (E) 2321-1822 | ISSN (P) 2321-8975

https://www.ijmhr.org/ijpr.html

DOI-Prefix: https://dx.doi.org/10.16965/ijpr

\section{Article Information}

Received: 09 Apr 2020

Peer Review: 13 Apr 2020

Revised: None
Accepted: 15 May 2020

Published (O): 11 Jun 2020

Published (P): 11 Jun 2020

\section{INTRODUCTION}

Stroke or brain attack is the sudden loss of neurological function caused by an interruption of the blood flow to the brain [1]. The lower limb weakness comprises of $48.6 \%$ of the common impairments seen in stroke survivors. Disturbance in balance after stroke is a major concern which increases the level of dependency for activities of daily living and increases the risk of fall. Balanceimpairmentafter stroke existduring self-initiating movement and while reacting to destabilizing external force.
Belgen et. al, reported that for individuals with chronic stroke, history of falls was associated with fall-related self-efficacy, fear of falling, and depressivesymptoms [2]. Studies of balance impairments consistently have shown that people with stroke have greater postural sway than age matched healthy volunteers [3-7], also have altered weight distribution patterns, and smaller excursion when moving their weight around the base of support, especially in the direction of the weaker leg [8].

In order to improve balance abilities, many 
studies have adopted wide range of training methods involving, mental practice for rehabilitation of stroke patients have been reported [9-11].

Motor imagery also known as mental practice is defined as a dynamic state during which a subject mentally simulates a given action.

Motor imagery can also be defined as the covert cognitive process of imagining a movement of your own body (body part) without actually moving that body (body part) [12].

Motor imagery is a cognitive operation that increases brain activity in neuronal cortical networks $[13,14]$. Repetition and practice of motor scenes and routines by imagery is known to facilitate the learning or relearning of motor tasks [15]. Motor training performed by using imagery is a low-cost and low-risk motor rehabilitation intervention for post stroke individuals [16-19].The justification for using motor imagery as a rehabilitation tool is supported by brain-imaging studies showing that comparable brain areas are activated during actual performance and during mental rehearsal of the same tasks [20-23]. It is further strengthened by studies of motor imagery pointing to the reorganization of functional neuronal networks in healthy subjects and people post stroke [24].

Motor imagery involves rehearsing a known motor act without any visible muscle contraction or motor output. Numerous studies have indicated that motor imagery result in the same plastic changes in the motor system as actual physical practice [25].

Nowadays in most rehabilitation protocol mental practice is being implemented. The repetitive activation of motor system by mental practice is found to enhance motor performance \& muscle strength. However, prolonged mental practice also induced mental fatigue.

Studies have shown, prolonged physical practice as well as prolonged mental practice induces physical and mental fatigue respectively. An excessive physical practice can lead to muscular fatigue. In a recent study, by Rozand et. al. (2015) subject perceived greater mental fatigue after 20 minute training session of 80 maximal imagined contractions of elbow flexors [26]. During mental practice of fine motor skills, Gentili et. al, observed that subject started to develop fatigue and lose concentration after 60 trials of imagined pointing movements [27]. As their goal was to analyze the improvement of motor performance following mental compared to physical practice, they limited theirtraining to 60 trials. Mental practice of fine motor skills seems to be more sensitive to training, as one training session is sufficient to observe an improvement of motor performance, but could also be more sensitive to mental fatigue.

The combination of alternate mental and physical practice would help to overcome mental fatigue induced by prolonged mental practice. Also exercise session becomes more monotonous after particular repetition, thus we hypothesize if the sequencing combination of mental practice along with physical practice implemented in an exercise session would yield in different results.There are limited evidences on combination of physical practice and mental practice in improving balance in stroke patients. Therefore the study was conducted to compare whether two intervening combination of physical practice and mental practice could yield varied results, in improving balance in stroke patients.

\section{METHODS}

This experimental study was conducted on 30 subacute stroke patients in age group 45-60 years. Onset of stroke not more than six months and Berg balance score between 21 to 40 were included. Individuals having contractures or deformity in lower limb that could interfere with standing and cognitive deficit scoring $<26$ on MoCA, those with known cardiorespiratory ailments and other neurological deficits were excluded.

Materials used were four inch medium density foam, $10^{\circ}$ incline ramp, stair step of $15 \mathrm{~cm}$ height, two stacked shoe boxes, $2.5 \mathrm{Kg}$ weight cuff, firm chair with armrest, measuring tape and masking tape.

Procedure: Ethical committee approval was obtained from institutional research ethics committee.Detailed information was given regarding the study and a written informed 
consent was obtained.

Participants were screened for inclusion and exclusion criteria including Berg Balance scale and Montreal cognitive assessment.

All the participants were assessed for balance using BESTest score sheet at the start of the protocol and at the end of protocol. Participants were also asked to rating $A B C$ scale. Participants were given scale in vernacular language (Hindi, Marathi and English). To start with, the scale was explained to the participant, along with its rating from $0-100 \%$ was explained and then accordingly they were asked to rate each item of the scale.

The participants were randomly assigned to two groups Group A and Group B by using chit method. Boththe group received conventional therapy for 3 days in a week for 4 weeks that included: Upper extremity exercises- active assisted exercises of shoulder, elbow and wrist for 10 repetitions. Hand exercises- Pick up small objects like buttons, coins etc, crumpling sheet of paper into a ball, buttoning, placing hand on the table, and try to lift each finger one at a time off the table. Lower extremity exercisesactive assisted exercises of hip, knee and ankle for 10 repetitions. Stretching- stretching of tight muscles including tendoachilles, hamstring, quadriceps, biceps, finger flexors for $30 \mathrm{sec}$ hold and 3 repetitions

Along with these exercises, Mental Practice and Physical Practice was given for eight activities.The participant practiced mentally in sitting position with arm and back rested and eyes closed.

A total of 100 imagined movements and 15 physical practices were given. The activities were: Standing up on toes, One leg standing, Stepping in forward and backward direction and sideways, Reach out for an object on floor, Semi tandem standing and Heel to toe (tandem) walking for five steps.

The intervention was conducted in a silent environment to allow for the subject to concentrate for mental practice.

Initially the practice session was given for learning of mental practice and get adapt for the session. In practice session each movement and its sequence was explained, i.e. how to perform it.
While imagining movements, Participants were provided with pen and paper and was told to mark on paper after each imagined movement, so that numbers of imaginations were counted. Counting for each imagined movement was monitored by therapist. The sequence of practice for group A and group B are as given below;

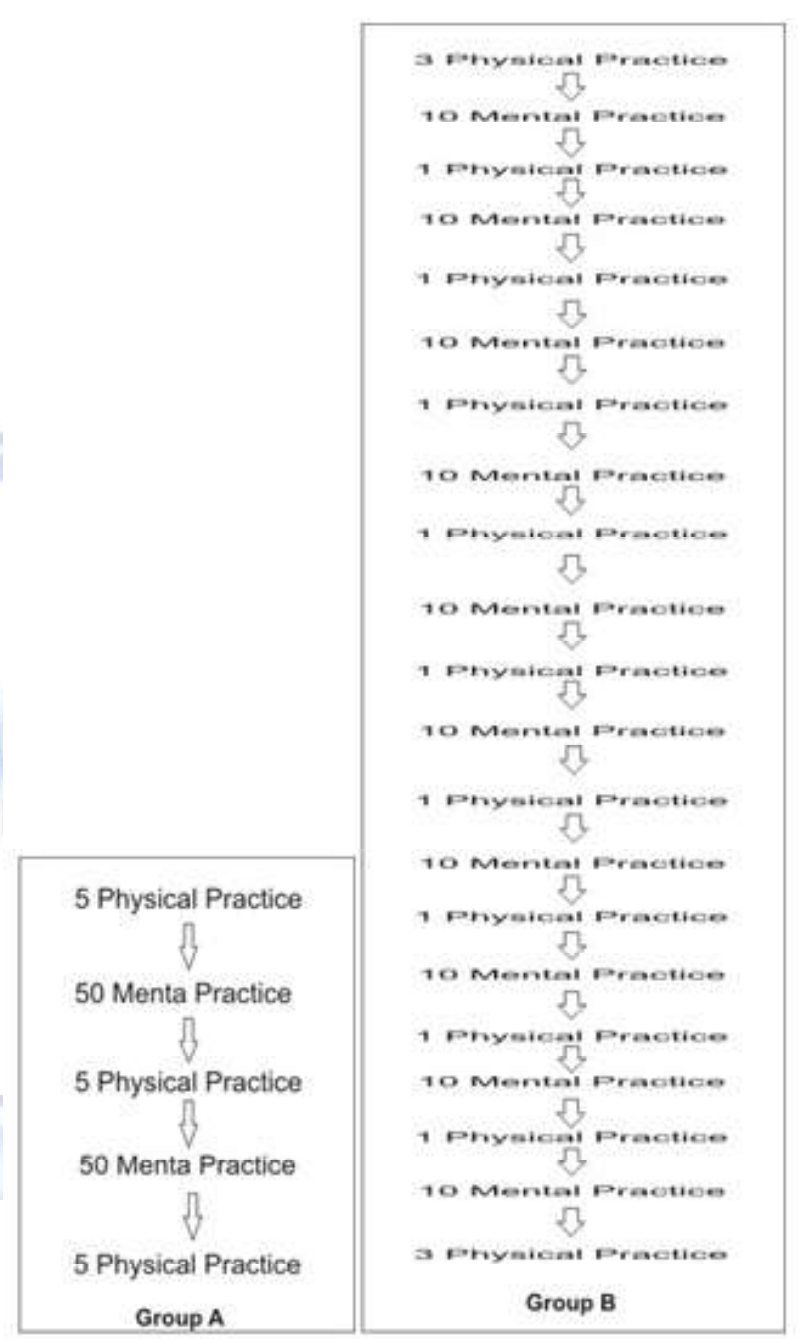

RESULTS

Statistical analysis: Data was initially explored to find out normal distribution by using WinPepi software.Statistical descriptive analysis was done by using Primer of Biostatistics software. Test of significance was set at $0.05(p<0.05)$.

Shapiro Wilk test was used for normal distribution. BESTest passed normality for group $A$ but not for group $B$, whereas $A B C$ scores passed normality for both groups. Thus for BESTest, paired $t$ test was used for within group analysis of group $A$ and Wilcoxon Signed Rank test for group B, whereas for between group analysis Mann-Whitney test was used as the baseline comparison was non-significant. 
Table 1:Age and Gender Distribution In Group A and Group B.

\begin{tabular}{|c|c|c|c|c|}
\hline \multicolumn{2}{|c|}{ Parameter } & Group A & Group B & Total \\
\hline Age & Mean+SD & $\mathbf{4 8 . 7 3 + 5 . 5 4}$ & $\mathbf{5 1 . 2 + 3 . 6 2}$ & - \\
\hline \multirow{2}{*}{ Gender } & Male & 11 & 12 & 23 \\
\cline { 2 - 5 } & Female & 4 & 3 & 7 \\
\hline
\end{tabular}

Table 2:Comparison of Change in Mean Score on Balance and Activities-Specific Balance Confidence Scalein Group A and Group B.

\begin{tabular}{|c|c|c|c|c|c|c|c|}
\hline $\begin{array}{l}\text { Outcome } \\
\text { Measure }\end{array}$ & Group & $\begin{array}{c}\text { Normal } \\
\text { Distribution }\end{array}$ & $\begin{array}{l}\text { Pretreatment } \\
\text { Mean+SD }\end{array}$ & $\begin{array}{c}\text { Postreatment } \\
\text { Mean+SD }\end{array}$ & Test Used & $\mathrm{T} / \mathrm{Z}$ value & $P$ value \\
\hline \multirow{4}{*}{ BESTest } & A & Yes & $31.65 \pm 1.50$ & $82.65 \pm 1.58$ & Paired t test & 80.863 & $<0.001$ \\
\hline & \multirow{2}{*}{ B } & \multirow{2}{*}{ No } & $29.75+4.04$ & $83.03+2.64$ & \multirow{2}{*}{$\begin{array}{c}\text { Wilcoxon Signed } \\
\text { Rank test }\end{array}$} & \multirow{2}{*}{3.396} & \multirow{2}{*}{$<0.001$} \\
\hline & & & Median- 30.56 & Median- 82.40 & & & \\
\hline & $A$ vs $B$ & & $\begin{array}{c}t=1.709 \\
p=0.099(N S)\end{array}$ & - & - & - & - \\
\hline \multirow{3}{*}{$A B C$} & A & Yes & $21.75+2.25$ & $23.38 \pm 2.13$ & Paired t test & 6.802 & $<0.001$ \\
\hline & B & Yes & $19.08 \pm 1.84$ & $20.78 \pm 2.01$ & Paired t test & 8.358 & $<0.001$ \\
\hline & $A$ vs $B$ & & $\begin{array}{c}t=3.603 \\
p=0.001(S)\end{array}$ & & the & - & - \\
\hline
\end{tabular}

Table 3:Comparison of Mean Difference (Post Minus Pre) of Balance and Activities- Specific Balance Confidence Scale Between Group A and Group B.

\begin{tabular}{|c|c|c|c|c|c|}
\hline $\begin{array}{l}\text { Outcome } \\
\text { Measure }\end{array}$ & Group & Mean士SD & Median & $\begin{array}{c}\text { Test } \\
\text { Applied }\end{array}$ & $t / z$ \\
\hline \multirow{2}{*}{ BESTest } & A & $51 \pm 2.443$ & 50.98 & \multirow{2}{*}{$\begin{array}{c}\text { Mann } \\
\text { Whitney }\end{array}$} & \multirow{2}{*}{$\begin{array}{c}Z \text { z sub T } \\
1.638 \\
p=0.101\end{array}$} \\
\hline & B & $53.29 \pm 4.446$ & 52.78 & & \\
\hline \multirow{2}{*}{$A B C$} & A & $1.5 \pm 1.07$ & - & \multirow{2}{*}{ ANCOVA } & \multirow{2}{*}{$\begin{array}{l}T=0.282, \\
p=0.780\end{array}$} \\
\hline & B & $1.717+0.79$ & - & & \\
\hline
\end{tabular}

Within group analysis of group $A$ and group $B$ for $A B C$ scores was done with paired test, whereas for between group analysis ANCOVA was used as the baseline comparison revealed significant difference.

\section{DISCUSSION}

Motor imagery training has the biggest advantage that, it is does not require physical exertion, since it is cognitive activity, so there is no limitation on the patient's ability to execute motions. Because of this advantage, motor imagery training is globally applied for a wide range of body functions.

There are evidences on motor imagery in improving balance in stroke patients withspecified duration of mental practice session. Jin Ho Chon et al, found significant improvement in balance ability of healthy subjects following 30 minutes of training session [28].

In this study, two sequencing combinations of physical practice and mental practice were formulated, since the need of our study was to avoid mental fatigue due to which physical performance could be affected.

With the first outcome measure BESTest analyzing pre and post scores, a significant improvement in the post scores was observed for both the groups.

The subjects reported physical fatigue in group $B$ due to frequent change of position and activity, which although we tried to overcome by 30 seconds rest in between the activities.

The second outcome measure Activities Specific Balance confidence, also showed improvement in post values. Statistically significant $p$ value of $<0.001$ was observed, but clinically not all the activities included in the scale showed improvement, i.e. activity like, stand on a chair and reach for something. This activity was not possible for the patient even after four weeks of the intervention program. Whereas activities like, walk around the house, reach for a small can off a shelf at eye level, stand on your tiptoes and reach 
for something above your-head were possible after the intervention. Likewise there were few other activities which were not possible for patient to do from $A B C$ scale. The total score prior the treatment showed low physical function of the individual and even after the treatment regimen of 4 weeks, their total score was in low physical function only.

When change in both groups was compared a non-significant difference was observed for $B E S T e s t$ and $A B C$ scores stating that both the sequencing combinations were equally effective. However clinically it was observed that subjects in group $B$ performed better, also their mean values were higher than group $A$. This result proved that both sequencing combination of Mental practice along with Physical Practice are equally effective in improving balance in stroke patients.

Throughtout life, in health and disease, human brain reorganizes itself, this is neuroplasticity which forms the basis for human learning and adaptation [29].

Motor imagery in stroke is useful due to enhanced cortico-cortical interactions which facilitate recovery. During motor imagery changes in network connectivity were observed most clearly i.e. plasticity occurred in cortical areas that were remote from the subcortical infarct. In contrast to interactions with the supplementary motor area, the interactions among other areas including the prefrontal cortex are enhanced. This increased connectivity may have facilitated recovery of motor function. The imbalance between primary motor cortices to a more physiological state can be driven by motor imagery training after stroke, thus improving motor function.

Ehrsson et al. [20] showed activation of specific limb-areas in the primary motor cortex with imagined movements. It was shown that imagined finger and tongue movements can be distinguished from imagined toe movements.

A study done by Sjoerd de Vries \&Moulder showed that motor imagery, observation and execution are closely related phenomena sharing neuralcontrol processes. Although differences exist between execution, observation and motor imagery, authors suggest the existence of a general system involved in the representation of our own bodily actions that plays a role in the control of actions as well as in imagination and observation [30]. It has been argued that mirror neurons may play a crucial role in this process [31].

Rizzolatti et.al in their study on group of cells in monkey's area F5 in the pre motor cortex, suggest that, mirror neurons are involved in the coding of the goal of actions. In other words, mirror neurons are related to intentional behavior and seem to be involved in understanding the actions of others [32]. Many researchers believe that mirror neurons may play a role in motor learning [33].

In the present study, as there was no statistical significant difference between both the groups $A$ and $B$, but the mean values for Group $B$ was slight better than group $A$, for the both the outcome measure BESTest and ABC scale. Supporting to our finding, the study done by Rozand et al (2015), in this study, participants imagined 100 point-to-point arm movements combined with actual pointing movements every 10 or 50 imagined movements. After imagining 100 pointing movements, subjective feeling of mental fatigue was reported by participants. At the end of the protocol the duration of both actual and imagined movements were increased, when participants performed actual movements every 50 imagined movements. On the contrary, no change in actual and imagined movement duration was observed when participants performed actual movements every 10 imagined movements. These results suggested that the repetition of many imagined movements induced mental fatigue and altered the mental simulation and the actual execution processes of the movement. However, the regular execution of actual movements seemed to counteract the negative effect of mental fatigue as both actual and imagined movement duration remained constant with actual trials inserted between mental rehearsals. So according to study it was suggested that during training or rehabilitation programs, actual movements should be executed and/or duration of movement should be controlled to avoid the negative effects of mental fatigue on motor performance. 
Motor Imagery does not require expensive technology, equipment, instrumented locations, and it does not physically exhaust the individual. Patient can practice Motor Imagery technique independent from the therapist, location, and time of the day after initial learning. Facet of Mental practice is particularly advantageous in stroke rehab since, certain motor skills may be difficult and unsafe to physically practice, making it useful to be able to approximate physical practice conditions. Since, Motor Imagery therapy does not require special equipment and can be easily performed at home by patients or their family members after receiving professional training. This therapy also increases patients' functional status, encourages participation, facilitates continuous treatment and care, and reduces financial burden of stroke patients with long-term rehabilitation; thus, $\mathrm{MI}$ therapy is both cost effective and clinically effective

In our study, as we got improvement in both the groups, irrespective of any sequence in combination of Mental practice and Physical practice it is clear that improvement is due to repetition of Mental practice added to Physical practice, and not any particular sequence. So Mental practice and Physical practice can be given to the patient irrespective of any specific sequence. The treatment regimen can be formulated considering the patients physical condition, whether in acute, sub-acute or chronic stage and fatigability. Special care must be taken while selecting patient, especially patient with low cognitive level, since Motor imagery is complex cognitive process. $\mathrm{MI}$ is emerging as a beneficial technique used in combination with routine rehabilitation training in patients after stroke, and no serious adverse events were noted during MI therapy.

\section{Conflicts of interest: None}

\section{REFERENCES}

[1]. Susan B. O'Sullivan, Thomas J. Schmitz, Physical Rehabilitation, FifthEdition.

[2]. Belgan B, Beninato $M$, Sullivan PE, Narielwalla K: The association of balance capacity and falla self efffecacy with history of falling in community-dwelling people with chronic stroke. Arch Physc Med Rehabil. 2006;87(4):554-561.
[3]. Corriveau H, Hebert R, Raiche M, Prince F. Evaluation of postural stability in the elderly with stroke. Arch Phys Med Rehabil. 2004;85:1095-1101.

[4]. De Haart M, Geurts A, Huidekoper S, et al. Recovery of standing balance in post-acute stroke patients: a rehabilitation cohort study.Arch Phys Med Rehabil. 2004;85:886-895.

[5]. Lamontagne A, Paquet N, Fung J. Postural adjustments to voluntary head motions during standing are modified following stroke. Clin Biomech. 2003; 18: 832- 842 .

[6]. Ikai T, Kamikubo T, Takehara I, et al. Dynamic postural control of patients with hemiparesis. Am J Phys Med Rehabil. 2003;82:463-469.

[7]. Mizrahi J, Solzi R, Ring H, Nisell R. Postural stability in stroke patients: vectorial expression of asymmetry, sway, activity and relative sequence of reactive forces. Med Biol EngComput. 1989;27:181-190.

[8]. Sarah F Tyson, Marie Hanley et al, Balance Disability After Stroke, Physical Therapy, 2006;86(1).

[9]. Liu H, Song L, Zhang T: Changes in brain activation in stroke patients after mental practice and physical exercise: a functional MRI study. Neural Regen Res, 2014;9: 1474-1484.

[10]. Oostra KM, Oomen A, Vanderstraeten G, et al. Influence of motor imagery training on gait rehabilitation in sub-acute stroke: a randomized controlled trial. J Rehabil Med, 2015, 47: 204-209.

[11]. Cho HY, Kim JS, and Lee GC: Effects of motor imagery training on balance and gait abilities in post-stroke patients: a randomized controlled trial. Clin Rehabil, 2013, 27: 675-680.

[12]. Feltz, D.L. and Landers, D.M., The effects of mental practice on motor skill learning and performance: meta-analysis, J. Sport Psychol, (1983) 25-57

[13]. Cicinelli P, Marconi B, Zaccagnini M, Pasqualetti P, Filippi MM, Rossini PM. Imagery-induced cortical excitability changes in stroke: a transcranial magnetic stimulation study. Cereb Cortex 2006;16:247-53.

[14]. Kosslyn SM, Ganis G, Thompson WL. Neural foundations of imagery. Nat Rev Neurosci2001;2: 635-42.

[15]. Lafleur MF, Jackson PL, Malouin F, Richards CL, Evans AC, Doyon J. Motor learning produces parallel dynamic functional changes during the execution and imagination of sequential footmovements. Neuroimage 2002;16:142-57.

[16]. Braun SM, Beurskens AJ, Borm PJ, Schack T, Wade DT. The effects of mental practice in stroke rehabilitation: a systematic review. Arch Phys Med Rehabil2006;87:842-52.

[17]. Dickstein R, Deutsch JE. Motor imagery in physical therapist practice. Phys Ther2007;87:942-53.

[18]. Page SJ, Levine P, Leonard A. Mental practice in chronic stroke: results of a randomized, placebocontrolled trial. Stroke 2007;38: 1293-7.

[19]. Sharma N, Pomeroy VM, Baron JC. Motor imagery: a backdoor to the motor system after stroke? Stroke 2006;37:1941-52. 

PRACTICE ON BALANCE IN STROKE PATIENTS.

[20]. Ehrsson HH, Geyer S, Naito E. Imagery of voluntary movement of fingers, toes, and tongue activates corresponding body-partspecific motor representations. J Neurophysiol2003;90:3304-16.

[21]. Jeannerod M, Decety J. Mental motor imagery: a window into the representational stages of action. CurrOpinNeurobiol 1995;5: 727-32.

[22]. Jeannerod M, Frak V. Mental imaging of motor activity in humans. CurrOpinNeurobiol 1999;9:7359.

[23]. Mulder T, de Vries S, Zijlstra S. Observation, imagination and execution of an effortful movement: more evidence for a central explanation of motor imagery. Exp Brain Res 2005; 163: 344-51.

[24]. Johnson-Frey SH. Stimulation through simulation? Motor imagery and functional reorganization in hemiplegic stroke patients. Brain Cogn 2004; 55: 328-31.

[25]. JagunTomar et.al. Comparative Study of Mental Imagery, Embedded Mental Imagery and Balance Training in Improving Balance Control Ability in Geriatric Population, European Academic Research2014;II(9).

[26]. Vianney Rozand et.al, Prolonged motor imagery sessions alter imagined and actual movement durations: potential implications for neuro-rehabilitation, Behavioural Brain Research September 2015.

[27]. Gentili R, et al. Motor learning without doing: trialby-trial improvement in motor performance during mental training. Neurophysiology 2010.
[28]. Jin Ho Chon et al, Effects of Mental training on the balance control ability of healthy subjects, Department of Physical Therapy, GanhneungYeongdong College, J. Physc. Ther. Sci. 2010.

[29]. Wolpert D, Ghahramani Z. Computational principles of movement neuroscience. Nat Neurosci. 2000; 3 (suppl): 1212-1217.

[30]. Sjoerd de Vries and Theo Mulder, Motor Imagery And Stroke Rehabilitation: A Critical Discussion, Centre for Human Movement Sciences, University Medical Centre Groningen, University of Groningen, Groningen and Royal Netherlands Academy of Arts and Sciences, Amsterdam, The Netherlands, J Rehabil Med 2007;39:5-13.

[31]. Rizzolatti G. The mirror neuron system and imitation. In: Hurley S, Chater N, eds. Perspectives on imitation: from cognitive neuroscience to social science. Cambridge, MA: MIT Press; 2005, p. 55-77.

[32]. Rizzolatti G, Gentilucci M, Fogassi L, Luppino G, Matelli M, Ponzoni- Maggi S. Neurons related to goal- directed motor acts in inferior area 6 of the macaque monkey. Exp Brain Res 1987;67: 220-224.

[33]. Gallese V. Embodied simulation; from neurons to phenomenal experience. Phenomenal Cognitive Sci 2005; $4: 23-48$.

How to cite this article:

Neha Ramchandra Tambe, Shamla WamanraoPazare. EFFECT OF TWO SEQUENCING COMBINATIONS OF PHYSICAL PRACTICE AND MENTAL PRACTICE ON BALANCE IN STROKE PATIENTS. Int J Physiother Res 2020;8(3):3471-3477. DOI: 10.16965/ijpr.2020.131 